Planetary and Space Science, 2007, in press

\title{
LUNAR DUST CHARGING BY PHOTOELECTRIC EMISSIONS
}

M. M. Abbas ${ }^{1}$, D. Tankosic ${ }^{2}$, P. D. Craven ${ }^{1}$, J. F. Spann ${ }^{1}$ A. LeClair ${ }^{2}$, and E. A. West ${ }^{1}$.

${ }^{1}$ NASA-Marshall Space Flight Center, Huntsville, AL 35812

${ }^{2}$ University of Alabama in Huntsville, Huntsville, AL 35899 


\begin{abstract}
The lunar surface is covered with a thick layer of sub-micron/micron size dust grains formed by meteoritic impact over billions of years. The fine dust grains are levitated and transported on the lunar surface, as indicated by the transient dust clouds observed over the lunar horizon during the Apollo 17 mission. Theoretical models suggest that the dust grains on the lunar surface are charged by the solar UV radiation as well as the solar wind. Even without any physical activity, the dust grains are levitated by electrostatic fields and transported away from the surface in the near vacuum environment of the Moon. The current dust charging and levitation models, however, do not fully explain the observed phenomena. Since the abundance of dust on the Moon's surface with its observed adhesive characteristics has the potential of severe impact on human habitat and operations and lifetime of a variety of equipment, it is necessary to investigate the charging properties and the lunar dust phenomena in order to develop appropriate mitigating strategies.

Photoelectric emission induced by the solar UV radiation with photon energies higher than the work function of the grain materials is recognized to be the dominant process for charging of the lunar dust, and requires measurements of the photoelectric yields to determine the charging and equilibrium potentials of individual dust grains. In this paper, we present the first laboratory measurements of the photoelectric efficiencies and yields of individual submicron/micron size dust grains selected from sample returns of Apollo 17, and Luna 24 missions, as well as similar size dust grains from the JSC-1 simulants. The measurements were made on a laboratory facility based on an electrodynamic balance that permits a variety of experiments to be conducted on individual sub-micron/micron size dust grains in simulated space environments. The photoelectric emission measurements indicate grain size dependence
\end{abstract}


with the yield increasing by an order of magnitude for grains of sub-micron to several micron size radii, at which it reaches asymptotic values. The yield for large size grains is found to be more than an order of magnitude higher than the bulk measurements on lunar fines reported in the literature.

Key words: Lunar dust, dust charging, photoelectric emission, photoelectric yield, lunar dust levitation.

\section{INTRODUCTION}

Ever since the astronauts' surprising observations of the ubiquitous nature of dust in the lunar environment during the Apollo missions, a great deal of theoretical and experimental activity has been devoted to investigate the properties of lunar dust and the nature of the observed physical phenomena. The astronauts found the lunar dust to be unexpectedly high in its adhesive characteristics, sticking to the suits, the instruments, and the lunar rover. Lunar Surveyor Spacecrafts (Criswell, 1973; Rennilson and Criswell, 1974) and the Lunar Ejecta \& Meteorite Experiment on Apollo 17 (Berg, 1978) indicated the presence of transient dust clouds in the lunar environment. A horizon glow over the lunar terminator and high altitude streamers were observed by the astronauts on the Apollo 17 spacecraft (e. g., Criswell, 1973, McCoy and Criswell, 1974, Rennilson and Criswell, 1974; Pelizzari and Criswell, 1978). This phenomenon was observed during the lunar sunrise and sunset by astronauts both on the surface and in the spacecraft in orbit, and was recorded in their logbooks. The Clementine Spacecraft in a later mission has also detected the phenomenon of high altitude lunar horizon glow (Zook and McCoy, 1991; Zook et. al., 1995).

The lunar regolith, formed by the impact of meteorites, micrometeorites, cosmic rays, and the solar wind over billions of years, is composed of irregularly shaped fine and coarse dust 
grains with size distribution in the range of nano-meter, sub-micron, centimeter size or larger. The lunar gravity is about $1 / 6^{\text {th }}$ of the earth and surface pressure is $10^{-12}$ torr $\left(\sim 2 \times 10^{5}\right.$ $\mathrm{mol} / \mathrm{cm}^{-3}$ ). With virtually no atmosphere, the lunar regolith is exposed to the intense unimpeded solar electromagnetic radiation in the visible, UV, and x-ray spectral regions, as well as charged particle radiation that includes high energy (1-10 GeV/nucleon) galactic cosmic rays, and the solar wind flux. In addition, even the smallest micrometeorites impact the lunar surface at high velocities. The lunar samples returned by the Apollo and Luna missions indicate $20 \mathrm{wt} \%$ of the regolith to be $<20 \mu \mathrm{m}$ in size and $\sim 10 \mathrm{wt} \%<10 \mu \mathrm{m}$ in size, with a smaller fraction consisting of sub-micron size grains. The chemical composition of the lunar soil varies considerably between the maria and the highlands. Measurements by Apollo 11 to 17 missions indicate significant variations in composition at different sites with major constituents in the soil consisting of $\mathrm{SiO}_{2}, \mathrm{AlO}_{3}, \mathrm{FeO}$, and other constituents with smaller percentages (e.g., Carrier, III, W D., 1973; Johnson et al., 1972; McKay et al., 1974; Rose et al., 1975; Heiken at al., 1991).

The solar wind, UV radiation, X-rays, and cosmic rays, lead to charging of the lunar regolith and the dust grains. The main processes by which the lunar regolith and the dust grains may be charged include: (a) Electron or ion collisions on the night side, generally leading to negatively charged grains with low energy $(<100 \mathrm{eV})$ electrons. Solar wind electrons with sufficiently high energy may produce positively charged grains by the secondary electron emission process. (b) Charging by photoelectric emissions by photons with energies higher than the work function of the grain material. (c) Triboelectric charging of dust grains by contact charging process in which electrons are transferred from a solid material with high work function to one with a lower work function. The complex dust phenomena observed on the 
lunar surface is attributed to electrostatic charging of the lunar regolith with the distribution of small sub-micron/micron size particles in the radiation environment, and the like charges repelling each other. The dust grains are levitated and transported in the vertical and horizontal directions by the electric fields established by the dust and the ambient plasma. The dominant charging process on the dayside of the lunar surface is believed to be the UV radiation at wavelengths $<200 \mathrm{~nm}$ with photon energies higher than the work function of the lunar regolith materials. On the night side, the interaction of the solar wind plasma with the lunar regolith, charges the dust grains negatively, predominantly by low energy electrons due to their higher mobility (Halekas et al, 2002). The large electric fields created over the terminator are assumed to produce the dust clouds that are observed as a glow produced by sunlight scattered over the horizon. The current dust charging and levitation models, however, are only qualitative and do not fully explain the observed phenomena (Manka, 1973; Freeman et al., 1973; Pelizzari and Criswell, 1978; e.g., Horanyi, 1996; Horanyi, et al. 1995, 1998; Stubbs et al., 2005)

Since the abundance of dust on the Moon's surface with its observed adhesive characteristics is believed to have the potential of a severe deleterious effect on the human habitat, the operations, and the lifetimes of a variety of equipment in the projected exploration initiative, the lunar dust properties and the observed phenomena need to be investigated. Viable models of charging, levitation, and transportation of dust grains in the lunar environment need to be developed in order to implement effective mitigating strategies. A crucial parameter in evaluation of the dust charging mechanism is the photoelectric yield of sub-micron/micron size dust grains exposed to UV radiation, usually defined as the number of electrons emitted per photon absorbed. Accurate theoretical models for calculation of the photoelectric yields are not yet available, and information for photoelectric emissions for materials of various 
compositions has to be determined experimentally. Such measurements, however, are generally available for bulk materials only, although on theoretical considerations it has long been recognized that the photoelectric yields of individual sub-micron/micron size grains are substantially different from the corresponding values for the bulk materials (e.g., Watson, 1972; Gallo and Lama; Draine, 1978; de Heer, 1993; Wong et al., 2003). A laboratory facility that permits investigation of the optical and physical properties of individual micron size dust grains in simulated space environments has been developed at NASA Marshall Space Flight Center. This facility has been employed for measurements of: (a) Radiation pressure on individual micron size $\mathrm{SiO}_{2}$ spherical dust grains of $\sim 0.5$ to $3.4 \mu \mathrm{m}$ radii illuminated by $5320 \AA$ laser light (Abbas et al., 2003); (b) Rotation and alignment of individual SiC irregular shaped dust grains of effective radii of $\sim 0.1$ to $8 \mu \mathrm{m}$ radii exposed to $5320 \AA$ laser light simulating the rotation of dust grains in the interstellar medium (Abbas et al., 2004); (c) Photoelectric efficiencies and yields of individual dust grains of $\sim 0.1$ to $10 \mu \mathrm{m}$ radii of interstellar composition (Abbas et al., 2006a). The first photoelectric emission measurements on the analogs of individual interstellar dust grains reported in the above reference indicated the yields decreasing substantially with grain size for sub-micron size grains. This trend is consistent with the semi-classical models developed for atomic clusters as well as laboratory measurements on clusters composed of a few to several thousand atoms.

In this paper, we present the first measurements of the photoelectric efficiencies and yields of sub-micron/micron size individual lunar dust grains selected from sample returns of the Apollo 17 and Soviet Luna 24 missions. Measurements on similar size grains selected from the JSC-1 lunar simulants are also presented for comparison. Preliminary yield measurements on lunar dust grains have been given in previous short publications (Abbas et al., 2006a-c). A 
brief description of the experimental technique based on an electrodynamic balance employed in the photoelectric yield measurements is given in section 2 . The basic theory and the equations used for calculations of the photoelectric efficiency and yields of individual dust grains are discussed in section 3 . In section 4 we present the experimental results of the photoelectric efficiencies and yields of individual dust grains selected from the lunar sample returns of Apollo 17 and Luna 24 missions, and the JSC-1 lunar simulants. A theoretical discussion on the experimental results on photoelectric yields is given in section 5 , with a summary and conclusions in section 6.

\section{EXPERIMENTAL TECHNIQUE}

The photoelectric emission measurements on lunar dust grains were made on an experimental facility based on an electrodynamic balance that permits levitation of individual sub-micron /micron size particles in simulated space environments. The basic theory of the experimental technique has been given in detail in previous publications (e.g., Davis, 1985; Spann et al., 2001, Abbas et al., 2002). A brief description of the facility for measurements on lunar dust grains is given here for convenience.

\subsection{Experimental Equipment}

The electrodynamic balance employed here consists of top and bottom electrodes of hemispherical configuration kept at DC potentials with respect to the ground to balance the gravitational force on the dust grain, and a central electrode of cylindrical configuration at a high AC voltage. The applied fields produce a null potential at the geometric center of the system, and a charged grain injected into the chamber is trapped in the potential well. Mathematical solutions of the equation of motion of a particle in the electric field of such a configuration permit stable solutions only for a certain range of values of the AC and DC potentials and the AC frequency. A charged particle injected into such an electrodynamic 
balance may be kept stably trapped for extended periods of time and a variety of experiments may be conducted in simulated space environments. A fundamental quantity measured directly on the electrodynamic balance is the charge-to-mass ratio of the trapped particle and is given by

$$
\frac{q}{m}=\frac{g z_{o}}{C_{o} V_{d c}}
$$

where $g$ is the gravitational acceleration, $C_{0}$ is a geometric constant of the trap experimentally determined to be 0.68 , and $z_{0}=0.765 \mathrm{~cm}$ is the DC electrode distance to the trap center. With measurements of $V_{d c}$, the charge-to-mass ratio of a trapped particle is thus a directly measurable quantity and is the basis of all measurements on an electrodynamic balance. The mass $m$ and the charge $q$ of the trapped particle are determined separately with measurement of the effective diameter $\mathrm{D}$ of the particle of known mass density $\rho$.

A schematic of the experimental setup for photoelectric emission measurements on lunar dust grains is shown in Fig.1. The main components in the apparatus are: (a) An Electrodynamic balance (b) Electric power supplies: AC voltage source $\left(V_{a c} \sim 100-2000 \mathrm{~V}, f_{a c} \sim\right.$ 10-1000 Hz), a low DC voltage source $\left(V_{d c} \sim 0.01\right.$ to $\left.50 \mathrm{~V}\right)$, and a high DC voltage source $\left(V_{h} \sim\right.$ 0-1000V) for inductive charging of the dust particles. (c) Particle injector: A pressure impulse device for injecting positively/negatively charged particles (d) Particle imaging system: A HeNe laser based optical magnifying system to image levitated particles on a monitor (e) Vacuum system with pressure gauges for evacuation of the system to pressures $\sim 10^{-4}$ to $10^{-5}$ torr (f) Ultraviolet source based on a Deuterium lamp with a $\mathrm{MgF}_{2}$ window, a vacuum Monochromator with FWHM resolution of $8 \mathrm{~nm}$, and an optical system to limit the beam diameter $~ 3.5-4.5$ mm (FWHM) size, smaller than the entry/exit apertures of $\sim 6 \mathrm{~mm}$ in the balance ring electrode thus minimizing any secondary electron emission from the walls excited by any stray UV 
radiation (g) Photomultiplier tube (PMT) with a spectral response in the $115-200 \mathrm{~nm}$ wavelength region.

With some modifications, this facility has been employed for unique measurements of radiation pressure, rotation, and photoelectric efficiency of individual micron size dust grains, as mentioned in section 1. Preliminary measurements of charging properties of individual submicron/micron size dust grains selected from the sample returns of Luna 24 and Apollo 17 lunar missions have also been given in recent short publications (Abbas et al., 2006a,b).

\subsection{Experimental Procedure}

A small sample of each category of dust grains is mixed in distilled water, and a few drops of this uniformly mixed solution are inserted into a liquid particle injector. The particles are inductively charged with a high electric field in the particle injector fitted with a calibrated 10-50 $\mu$ m diameter orifice at the center (Spann, et al., 2001, Abbas et al., 2003). Although dry particle injections into the trap have also been attempted successfully, a wet particle injection method was used in this study for convenience, where all particles are initially charged negatively, and discharged by exposure to UV radiation. With suitable choice of the electrical potentials and the frequency, a single particle is stably trapped in the electrodynamic balance for extended periods of time. With wet particle injection, the dust grain loses the water content quickly, in particular after initiation of the evacuation procedure, and with the heating arising from exposure to the He-Ne laser light.

After stable trapping of an individual dust grain of known composition and density, the effective diameter of the grain is determined by utilizing the marginal stability conditions based on the atmospheric drag at ambient pressures of $\sim 1$ to 10 torr (e.g., Davis, 1985). With the measurement of the charge-to-mass ratio $q / m$ (Eq. 1), and the effective diameter of the particle 
of known composition and density $\rho$, the trapped particle is characterized both in terms of the charge $q$ and mass $m$. The pressure in the chamber is allowed to reach lower values of $\sim 10^{-4}$ to $10^{-5}$ torr for carrying out photoelectric emission experiments. With dust grains of known composition, density, and effective diameter, levitated in simulated space conditions, a variety of experiments may be conducted. An example of charging and discharging of a dust grain as a function of time is shown in Fig. 2 with a selected portion of the data, to illustrate the capability of the experimental facility. The plot shows a $2.3 \mu \mathrm{m}$ radius polystyrene particle with an initial charge of $\sim 885$ e, acquiring an equilibrium charge of $\sim 1280$ e by photoelectric emissions with UV radiation at $160 \mathrm{~nm}$ wavelength, discharged to a charge of $\sim 880$ e when exposed to a 2500 $\mathrm{eV}$ electron beam, and again reaching an equilibrium charge of $\sim 1287 \mathrm{e}$ when exposed to "white light” with radiation all wavelengths from the deuterium lamp at t $~ 1388$ min. Figure 3 exhibits the discharging of $0.18 \mu \mathrm{m}$ radius Apollo 17 negatively charged dust grain when exposed to UV radiation at wavelengths of $120 \mathrm{~nm}, 140 \mathrm{~nm}$, and $160 \mathrm{~nm}$. In about 75 minutes of exposure to the incident UV photons, the particle is discharged from an initial value of $~$ $180 e$ to less than 10e, at which it is ejected from the trap. The corresponding DC voltage required to keep the particle stable trapped at the balance center is also shown.

In the following section, we discuss the basic equations required in calculations of the photoelectric efficiency and yield of micron size dust grains by photoelectric emissions only.

\section{BASIC EQUATIONS FOR PHOTOELECTRIC EMISSIONS}

The experimental setup described above provides a straightforward capability for measurements of the photoelectric yields of individual dust grains levitated in the trap. The calculation of the photoelectric yield is based on direct measurements of the photoelectrons emitted per second by a dust grain exposed to UV radiation, and the number of photons 
incident on the dust grain per second. The former quantity is determined from Eq. (1) by monitoring the grain charge $q(t)$, and the discharge rate $\partial q / \partial t$, and the latter is calculated by measuring the photon flux in the UV beam by reflecting it to a photo-multiplier tube (PMT) and measuring the collected current. The number of photoelectrons emitted per second by a dust grain is a function of the charge or the surface potential of the grain and may be written as

$$
n_{d}^{e}\left(\phi_{s}\right)=\frac{i_{d}}{e}=\frac{1}{e} \frac{\partial q}{\partial t}
$$

where $\phi_{s}=q / 4 \pi \varepsilon_{0} a$, is the surface potential of the grain of effective radius $a$, $\varepsilon_{0}$ is the permittivity of free space, $i_{d}$ represents the current into the dust grain and $e$ is the primary electron charge. As discussed later, the discharge rate may be determined quite accurately, with the precision approaching the detection of a single electron emission at a time in sufficiently low charge regimes. In general, the accuracy in measurement of the absolute value of this quantity is limited only by the uncertainty in the measurement of the DC voltage $V_{d c}$ in Eq. (1), the effective diameter, and the trap constants.

The number of photons $N_{B}^{p h}$ per second in a UV radiation beam at wavelength $\lambda$ is determined with measurement of the current collected by a PMT with the projected cathode area larger than the UV beam width, and is given by:

$$
N_{B}^{p h}=\frac{i_{p m t}(\lambda)}{e \eta_{q}(\lambda) G \mathrm{R}}
$$

where $i_{p m t}(\lambda)$ is the current measured by the PMT at wavelength $\lambda, \eta_{q}$ is the quantum efficiency, $G$ is the gain of the PMT, and $R$ represents the reflectance of the mirror (Fig. 1). To 
evaluate the number of photons per second incident on a dust grain of effective diameter $\mathrm{D}$, we assume a Gaussian radial distribution of photons in the beam as:

$$
n_{B}^{p h}=n_{\max }^{p h}(0) \exp \left(-r^{2} / r_{e}^{2}\right)
$$

where $r_{e}=w_{e} / 2$ represents the width of the beam where the photon flux density $n_{B}^{p h}$ is $1 / \mathrm{e}$ value of the maximum value $n_{\max }^{p h}(0)$ at the beam center. The above assumption implies that the total number of photons in the beam and measured by the PMT is represented by

$$
N_{B}^{p h}=\left(\pi r_{e}^{2}\right) n_{\max }^{p h}(0)
$$

With the beam width $w_{\mathrm{e}} \sim 3.5$ to $4.5 \mathrm{~mm}$ being much larger than the particle diameter $\mathrm{D}$, equations (3) and (5) may be used to calculate the number of photons per second incident on the dust with:

$$
n_{d}^{p h}=\frac{i_{p m t}(\lambda)}{e \eta_{q}(\lambda) G \mathrm{R}} \cdot \frac{D_{\mu m}^{2}}{w_{e}^{2}(\lambda)}
$$

For the PMT used in the photoelectric emission measurements discussed in this paper, the gain $G=600$ and $R=0.6$, equation (6) may be written as:

$$
n_{d}^{p h}=1.73 \times 10^{16} \frac{i_{p m t}(\lambda)}{\eta_{q}(\lambda)} \cdot \frac{D_{\mu m}^{2}}{w_{e}^{2}(\lambda)}
$$

In the measurements presented here, the photoelectric efficiency is defined as the number of electrons emitted by the dust grain per incident photon, and is a function of the charge $q$ or the grain surface potential $\phi_{s}$ :

$$
E_{p e}=\frac{n_{d}^{e}\left(\phi_{s}\right)}{n_{d}^{p h}}
$$


The photoelectric yield, on the other hand, is usually defined in the literature as photoelectrons emitted per photons absorbed with the grain potential approaching zero, and may be written as

$$
Y=E_{p e} / Q_{a b s}=\frac{n_{d}^{e}\left(\phi_{s} \rightarrow 0\right)}{n_{d}^{p h} \cdot Q_{a b s}}
$$

where $Q_{a b s}$ is the absorption efficiency of the grain and may be obtained by using Mie scattering theory calculations with known particle diameter and the complex refractive index. The photoelectric efficiency of individual dust grains may thus be calculated by equations (8) through direct measurements of the quantities $n_{d}^{e}$ and $n_{d}^{p h}$ expressed by equations (2) and (7).

An electron emitted from negatively charged grains, as in all experiments discussed in this paper, experiences a repulsive Coulomb force that is a function of the grain charge or its surface potential. This force is in addition to an attractive force due to an image potential force that is attractive regardless of the polarity of the dust grain charge. The photoelectric efficiency is thus expected to show an upward trend for heavily charged grains at high surface potentials compared with the values when the grain is close to being completely discharged. The photoelectric yield, however, is generally defined for neutral bulk materials with the surface potential approaching zero, as in equation (9). In the measurements presented here, the photoelectric yields are therefore based on the photoelectric efficiencies evaluated by leastsquares regression to represent the values corresponding to the surface potential $\phi_{S}$ or the grain charge $\mathrm{Z}$ approaching zero. The photoelectric yield is then determined by equation (9) with the absorption efficiency $Q_{a b s}$ calculated by using Mie theory for the known of the effective diameter and the complex refractive index of the grain. 
It should be emphasized here that the photoelectric efficiency, required for lunar dust charging calculations, is the directly measured quantity reported in this paper, and it includes the absorption efficiency $Q_{a b s}$ of the dust grains. The calculation of the photoelectric yield with its complex definition, however, requires the unknown value of $Q_{a b s}$ at the incident photon energy, and is a function of the effective diameter and the complex refractive index of the dust grain. The variability of $Q_{a b s}$ with complex refractive index is indicated by the Mie theory calculations in Fig. 4, as a function of the size parameter in the range of $x=(2 \pi a / \lambda) \sim 10$ to 20. The three plots indicate values of $Q_{a b s}$ for three different complex refractive indices $\mathrm{m}=\mathrm{m}_{\mathrm{r}}$ $-\mathrm{im}_{\mathrm{i}}$ : (1.9, -0.74) for $120 \mathrm{~nm}$ wavelength radiation, $(1.95,-0.55)$ at $140 \mathrm{~nm}$, and $(2.03,-0.39)$ at $160 \mathrm{~nm}$. The calculated absorption efficiency for above three cases respectively are: 1.10 , $1.12,1.13$ at the size parameter $x=10$; $Q_{a b s} \sim 0.98,1.0,1.0$, for $\mathrm{x}=20$; and $0.85,0.86,0.87$ for $\mathrm{x}=100$. The variation of $Q_{a b s}$ is thus of $\sim$ few percent over the range of size parameters considered in the measurements. It is larger for small values of $x$ corresponding to nanometer size particles not considered here.

\section{EXPERIMENTAL RESULTS ON PHOTOELECTRIC EFFICIENCIES AND YIELDS OF LUNAR DUST GRAINS AND LUNAR SIMULANTS}

Measurements of photoelectric efficiencies have been carried out on sub-micron/micron dust grains selected from the sample returns of Apollo 17 and Luna 24 missions, as well as the JSC-1 lunar simulants. The size distribution of the selected particles is in the range of $\sim 0.1$ to $12 \mu \mathrm{m}$ radii. The particles are exposed to a collimated UV radiation beam of width of $\sim 3.5$ to $4.5 \mathrm{~mm}$ at wavelengths of $120 \mathrm{~nm}, 140 \mathrm{~nm}$, and $160 \mathrm{~nm}$ (photon energies of 10.3 eV, $8.9 \mathrm{eV}$, 
and $7.8 \mathrm{eV}$ ). As the photoelectric efficiency is a function of the particle surface potential $\phi_{s}$, the measurements were made in cycles at the three wavelengths at various surface potentials, starting from the initial high value to a low value that approaches zero. The measured photoelectric efficiencies are generally found to be higher at higher surface potentials, with minimum values when the potential approaches zero. Since the photoelectric yield is defined for neutral particles as in Eq. (9), we adopt a simple procedure of plotting a least-squares linear regression to determine the efficiency that corresponds to the grain surface potential approaching zero. This value provides the photoelectric yield when convolved with absorption efficiency $Q_{a b s}^{\text {expt }}$ in accordance with Eq. (9). The absorption efficiency is calculated by using the Mie scattering theory (e.g., Bohren and Huffman, 1983, Wiscombe, 1979), for the measured effective radius of the particle determined by the "spring point" method. The grain mass density was also determined in the process of effective radius and drag evaluations, and was found to have about the same value as measured for all the Apollo mission's average value of $\sim 1.8$ g/cm ${ }^{-3}$ (e.g., Costes, N. C., 1972; Heiken, G. H., 1991). Calculated plots of $Q_{a b s}$ versus the size parameter $(2 \pi a / \lambda)$ are shown in figure (4) with the complex refractive index values for silica as in Draine and Lee (1984). These values for $Q_{a b s}$ have been used for evaluation of the photoelectric yields by equation (9) in the absence better experimental data, only to indicate the trend with grain size for comparison with the yield data for other materials published in the literature (Feuerbacher et al., 1972; Draine, 1978; Draine \& Lee, 1984; Müller et al., 1988). The basic experimental data presented in this paper, directly usable for evaluation of charging of lunar dust, are the photoelectric efficiencies of lunar dust grains over a range of the size parameters. With availability of more accurate experimental data for complex refractive 
indices and the corresponding values of $Q_{a b s}^{\exp t}$, the plotted yields may be corrected by multiplying the plotted values with the factor $\left(Q_{a b s} / Q_{a b s}^{\exp t}\right)$.

\subsection{Photoelectric Yields of Apollo 17 Lunar Dust Grains}

The lunar dust grains employed in the photoelectric measurements were selected from the finest portion of the sample soil ( $<20$ micron), Apollo 17, 71501, which is the sieved portion ( $<1 \mathrm{~mm}$ ) of the parent soil 71500. Photoelectric efficiency measurements were made on a number of negatively charged dust grains of effective radii in the 0.18 to $11.8 \mu \mathrm{m}$ range, at $\mathrm{UV}$ wavelengths of $120 \mathrm{~nm}, 140 \mathrm{~nm}$, and $160 \mathrm{~nm}$, at various surface potentials starting from the initial high value to a low value approaching zero when the particle is ejected from the trap. Figures 5a-c show the photoelectric efficiencies of three negatively charged Apollo 17 lunar grains of $0.18 \mu \mathrm{m}, 1.75 \mu \mathrm{m}$, and $3.2 \mu \mathrm{m}$ radii plotted as a function of surface potential with initial absolute values of $\sim 1.5 \mathrm{~V}, 40 \mathrm{~V}$ and $\sim 100 \mathrm{~V}$ respectively. The grains are discharged to potentials approaching zero at which they are ejected from the trap. The negatively charged grains generally indicate higher photoelectric efficiencies at higher potentials.

A composite plot of the photoelectric yields of all Apollo 17 particles vs. the size parameter $(2 \pi a / \lambda)$, employing $Q_{a b s}$ calculations using Mie theory (Fig. 4), is shown in Fig. 6. The only available measurements on bulk lunar fines by Feuerbacher et al., (1972) at the three corresponding wavelengths are also shown for comparison. The measurements made on individual dust grains indicate a strong size dependence of the yields for size parameters less than $\sim 100$, corresponding to particle radii of $\leq 1 \mu \mathrm{m}$ at wavelengths of $120-160 \mathrm{~nm}$ (photon energies of $6.7 \mathrm{eV}$ to $10.3 \mathrm{eV}$ ), decreasing by an order of magnitude for particles of smaller radii. For larger size particles, the yields approach constant asymptotic values, representing values corresponding to bulk materials. The asymptotic values for the three photon energies, 
however, are larger than the bulk measurements on lunar fines made by Feuerbacher et al., (1972) by factors of $\sim 14$ to 38 for the corresponding photon energies. Possible reasons for this discrepancy are discussed in section 5 . As discussed, the measured trend of the size dependence of higher yields for larger sub-micron size grains, although in conflict with the theoretical expectations of some earlier models, is found to be consistent with predictions of semi-classical models of the ionization potentials and work functions for atomic clusters, and experimental measurements on clusters composed of a few to several thousand atoms.

\subsection{Photoelectric Yields of Luna 24 Lunar Dust Grains}

The lunar dust samples returned by the Luna 24 Soviet mission were collected from Mare Cranium, on the far eastern edge of the Moon. Photoelectric emission measurements were made on a number of dust grains selected from the Luna 24 samples following a procedure similar to that discussed in the previous section for the Apollo 17 grains. Figures 7a,b show the plots of photoelectric efficiencies of dust grains of effective radii in the range $\sim 0.77 \mu \mathrm{m}$ to $3.38 \mu \mathrm{m}$ range, at three wavelengths of $120 \mathrm{~nm}, 140 \mathrm{~nm}$, and $160 \mathrm{~nm}(10.3 \mathrm{eV}, 8.9 \mathrm{eV}$, and 7.8 $\mathrm{eV}$ respectively). As for the Apollo 17 dust grains, the photoelectric efficiencies indicate a dependence on the surface potentials, with stronger dependence for the smaller grains. A composite plot of the photoelectric yields for 17 dust grains in the 0.1 to $3.38 \mu \mathrm{m}$ radii, randomly selected from the Luna-24 sample returns, is shown in Fig. 8 as a function of the size parameter for the three wavelengths of $120 \mathrm{~nm}, 140 \mathrm{~nm}$, and $160 \mathrm{~nm}$. Also shown for comparison are the measurements by Feuerbacher et al. (1972) made on bulk lunar fines for the three corresponding photon energies. 
Although Luna 24 grains were collected from different regions of the lunar surface, the yield measurements are found to be nearly the same as for the Apollo 17 grains for all three wavelengths, in asymptotic values as well as the trend with respect to the grain size.

\subsection{Photoelectric Yields of Dust Grains from JSC-1 Lunar Simulants}

The JSC-1 lunar simulants have been used for conducting a wide range of studies for investigating the lunar environment by simulating a variety of experiments required in preparation of the planned missions and human habitats on the Moon. The objectives for synthesizing a realistic simulant dust material are to replicate the lunar chemical composition with as many physical, chemical, and optical properties as closely as possible in an economical manner. However, the lunar samples are of a very much different nature in terms of the variability of physical configuration and the variability of grain elemental composition compared with the uniform composition of the JSC-1 simulants. With the lunar regolith and the fine dust having been formed over billions of years of impact of meteorites, micrometeorites, solar wind, energetic particles, and cosmic rays, it is nearly impossible to synthesize the lunar dust material in all its fine details. Therefore, for simulating specific dust properties that may influence a specific process under investigation, measurements on lunar dust simulants must be compared with measurements on lunar dust samples. For comparison of simulation of the charging properties by photoelectric emissions, we have carried out the photoelectric yield measurements on the JSC-1 lunar simulants, as well as on Apollo 17 or Luna 24 dust grains over similar size distributions. Since our measurements were made on randomly selected grains of various sizes, a comparison of the photoelectric emissions of the lunar grains with the simulants presented here thus represents only a comparison of the average charging process of grains of the two categories. 
Figures 9a, b represent the photoelectric efficiency measurements at UV wavelengths of $120 \mathrm{~nm}, 140 \mathrm{~nm}$, and $160 \mathrm{~nm}$, on two levitated JSC-1 simulant dust grains of $0.70 \mu \mathrm{m}$ and 3.25 $\mu \mathrm{m}$ radii, with the grains surface potentials with initial values of $\sim 11 \mathrm{~V}$ and $\sim 90 \mathrm{~V}$ respectively, to final values approaching zero. A composite plot of the photoelectric yields as a function of the size parameter, based on measurements on a number of dust grains with a size range of $0.17 \mu \mathrm{m}$ to $4.7 \mu \mathrm{m}$ radii is shown in Fig. 10, for the three photon energies of $10.3 \mathrm{eV}$, $8.9 \mathrm{eV}$, and $7.8 \mathrm{eV}(120 \mathrm{~nm}, 140 \mathrm{~nm}$, and $160 \mathrm{~nm})$. The yield plots are based on the photoelectric efficiency measurements corresponding to the value at surface potential approaching zero. As for the lunar dust grains, this value is determined for each particle by least-squares regression of the efficiency plots as a potential of the surface potential. The grain absorption efficiencies $Q_{a b s}$ are calculated by using the Mie scattering theory shown in the Fig. 4. As for the lunar grains, the asymptotic values of the photoelectric yields are substantially higher than the bulk measurements on lunar fines, with the functional dependence of the yields on dust size following the same trend as the lunar dust grains. Comparison of the asymptotic values for large size JSC-1 grains with the Apollo 17 or Luna 24, however, indicates lower values for the JSC-1 grains, with Apollo 17 to JSC-1 yield ratios of $\sim 1.5$ to 4 . 


\section{DISCUSSION}

\subsection{Possible sources of errors in measurements}

The experimental technique employed in measurements of the photoelectric efficiencies and yields on individual levitated dust grains in an electrodynamic balance, as in all experiments, is subject to measurement errors. It is important to examine the possible sources of errors that would systematically bias the results or introduce errors that would render the results and the conclusions invalid. A systematic study with analytical and experimental evaluations of possible sources of errors was carried out for similar measurements on the analogs of cosmic dust grains and has been discussed in detail in section 5 of Abbas et al. (2006). A brief summary is given here for convenience.

The accuracy of experimental determination of the photoelectric efficiency of levitated individual dust grains is based on the accuracy of measurements of the following basic quantities in accordance with equations (3-7), and any possible influence of external environmental effects:

(1) $n_{d}^{p h}$, the number of photons incident on a dust grain of diameter $D_{\mu m} ; N_{B}^{p h}$, the total number of photons in the UV beams of width $w_{e} \sim 3500-4500$, measured with a PMT as $i_{p m t}(\lambda)$. All of the quantities involved in evaluation of $n_{d}^{p h}$ are directly measurable with high accuracy

(2) $n_{d}^{e}$, defined by equation (2), involves the basic measurement of the grain charge as a function of time $\partial q / \partial t$, with accuracy of $q$ approaching detection of a single electron in certain particle size and low charge regimes.

(3) Effects of: scattered UV radiation, electron emission from the trap walls, collisions with the ambient gas, particle oscillations in the radiation beam, any systematic effect 
difference in particle shapes and surface features, electrical fields of the DC and high AC voltages. All of the possible sources were systematically investigated analytically as well as by diagnostic experiments, with the conclusion that are negligible and do not lead to any systematic biases in the measured data.

\subsection{Theoretical Considerations and comparison with measurements}

Basic theory and detailed discussions relating to charging of dust grains by photoelectric emissions are available in standard texts on solid-state physics (e.g., Cusack, 1958; Kittel, 1996). Briefly, the electrons at the surface of the neutral dust grains are confined to a potential well of the order of a few angstroms produced by equal and opposite charges believed to exist at the surface of a neutral grain. An excited electron moving towards the surface may be ejected from a grain if it has sufficient energy: to overcome the energy lost in inelastic scattering, to penetrate the double layer potential barrier at the surface, and to overcome the attractive force of the image potential of the ejected electron. An ejected electron from a positively charged particle $\mathrm{Ze}(\mathrm{Z}>0)$ is subjected to a strong attractive force due to the image potential at short distances of the order of $\sim 10-500 \AA$ from the surface, and an attractive Coulomb force at longer distances. For negatively charged particles $(\mathrm{Z}<0)$, an ejected electron experiences an attractive force at short distances but also a repulsive Coulomb force at larger distances. No rigorous theoretical models of the photoelectric efficiencies or yields of individual small dust grains are available as yet.

The laboratory measurements of the photoelectric yields of Apollo 17, Luna 24 lunar submicron/micron dust grains, and the JSC-1 simulants presented here have some surprising features in view of the size dependence and the significant discrepancy with the available data for bulk lunar fines. The size dependence of the yields is found to exhibit higher values for larger grains, increasing by more than an order of magnitude with the yields approaching asymptotic values for grains of larger than a few micron radii. The asymptotic values, that are expected to be comparable to the measurements on lunar bulk fines, are found to be higher by factors of $\sim 35$ at $120 \mathrm{~nm}$ and of $\sim 15$ at $160 \mathrm{~nm}$. The measured trend of the photoelectric 
yields reported here for the lunar dust grains is similar to that observed for analogs of interstellar type micron size dust grains and reported elsewhere with detailed discussion of the possible sources of measurement errors (Abbas et al., 2006a).

A rigorous theory for calculation of the photoelectric efficiencies and yields of small submicron/micron size particles is not available as yet, and a viable explanation for the measured trend is based only on the semi-classical models. The earlier models based on geometric considerations predicted a size dependence of the photoelectric yields of sub-micron sized particles higher than the bulk values with an enhancement factor that depends on the particle radius $a$, and the characteristic escape lengths $l_{e}$ and absorption lengths $l_{a}$ (e.g., Watson, 1972; Draine, 1978; Weingartner and Drains, 2001). However, there are no definitive measurements on individual dust grains to validate the size dependence predicted by this model. A set of measurements on an ensemble of silver and gold nano-size particles appeared to indicate an enhancement compared with the bulk values for the photoelectric emissions for the silver particles only, with no enhancement for the gold particles. However, it was discovered that the observed enhancement for the silver particles was only due to contaminations of the silver particles (Burtscher and Schmidt-Ott, 1982; Burtscher et al., 1984; Müller et al., 1988). Also, the photoelectric yields for small nano-size particles of $2.7 \mathrm{~nm}$, $3.8 \mathrm{~nm}$, and $5.8 \mathrm{~nm}$ radii exhibit size dependence proportional to the particle radius, with the yield for the $5.8 \mathrm{~nm}$ enhanced by a factor $\sim 2$ relative to the values for the $2.7 \mathrm{~nm}$ particles (Müller et al., 1988). This result is contrary to the trend predicted by the above model, but consistent with the measurements presented in this paper.

In addition to the above model employed for the photoelectric yields for astrophysical applications, a large body of literature exists on work functions, ionization potentials, or the 
ionization energies of aggregates or cluster of atoms (e.g., Gallo and Lama, 1974, 1976a,b; de Heer, 1993; Wong et al., 2003). Semi-classical models based on image potentials have been developed for application to atomic clusters of a few atoms to a large number of atoms that could be considered to be close to bulk materials. The basic premise behind the approach for development of these models is the expectation of a systematic transition of ionization potential (IP) for single atoms, to clusters of a few to large number of atoms, extending to work function (WF) for the bulk materials. These models are designed for understanding the evolution of the physical properties of atomic systems progressing to large clusters, with the ability to predict the energy required to remove an electron from a particle of arbitrary size. Atomic clusters composed of $\mathrm{N}$ atoms in the models are assumed to have radii $R_{c l} \propto N^{1 / 3}$, and the ionization potentials vary with $\mathrm{N}$ as $\propto N^{-1 / 3}$. The models are being tested and validated with new cluster production and measurement techniques on clusters consisting of a few atoms to thousands of atoms (e.g., Ekardt, 1985; Seidl et al., 1991; Bréchignac et al., 1992; Dugourd et al. 1992; de Heer et al., 2003; Wong et al. 2003). The measurements clearly indicate lower ionization potentials and therefore higher expected photoelectric yields for larger clusters. Specifically, the ionization cross-section measurements as a function of photon energy by Bréchignac et al. (1992) for alkali atom-clusters of 170, 500, and 900 atoms indicate some surprising features with successively much higher plateau values for the larger clusters. The size dependence of the photoelectric yield measurements on sub-micron/micron size lunar dust grains presented in this paper are thus consistent with the photoelectric yield, ionization potential and ionization cross-section measurements on clusters and nano-meter size particles. Other than the similar photoelectric emission measurements on the analogs of cosmic dust grains (Abbas et al., 2006), 
no direct measurements on individual sub-micron/micron size dust grains are available for comparison.

\subsection{Comparison with bulk measurements on lunar fines}

The only photoelectric emission measurements on lunar dust reported in the literature are those made by Feuerbacher et al., (1972) on Apollo 14 samples of lunar fines. The measurement technique was based on a lunar dust sample placed in a stainless steel cup of 1.5 cm diameter and illuminated by UV radiation at $50 \mathrm{~nm}$ to $200 \mathrm{~nm}$ wavelengths. The maximum yield of $\sim 0.07$ was found to occur at $\sim 90 \mathrm{~nm}$, rapidly falling off to lower values of $\sim 10^{-7}$ at wavelengths of $250 \mathrm{~nm}$. The yield values at $120 \mathrm{~nm}, 140 \mathrm{~nm}$, and $160 \mathrm{~nm}$, as indicated on the plot in Figs. 6, 8, and 10, are substantially lower than the asymptotic values reported in this paper, that may be assumed to represent the values for the bulk materials. An explanation for the discrepancy between the two measurements is not clear. However, it is important to note that the measurements by Feuerbacher et al. (1972) were made on an aggregate lunar sample composed of lunar fines of less than $1 \mathrm{~mm}$ in size, $20 \%$ by weight of which are believed to consist of smaller than $20 \mu \mathrm{m}$, and $10 \%$ by weight of smaller sizes. Since the UV photon absorption and electron ejection and escape is a complex process, the yield measurements by Feuerbacher et al. (1972) may not be truly representative of bulk measurement. It is not inconceivable that the measurements represent the average yield of sub-micron/micron size grains in the lunar sample under test. If the yields were assumed to represent the values for sub-micron size particles levitated above the sample surface in the stainless steel cup, the inferred particle size would be of $\sim 0.5-1 \mu \mathrm{m}$ diameters for yields comparable to those reported in this paper. 


\section{SUMMARY/CONCLUSIONS}

We have presented the first photoelectric efficiency measurements made on individual dust grains selected from the Apollo 17 and Luna 24 samples as well as the JSC-1 lunar simulants. A summary and significant results of the measurements are summarized in the following.

(1). The measurements were made on individual dust grains with effective radii in the 0.1 to $11.8 \mu \mathrm{m}$ range, levitated in an electrodynamic balance and illuminated with UV radiation at wavelengths of $120 \mathrm{~nm}, 140 \mathrm{~nm}$, and $160 \mathrm{~nm}(10.3 \mathrm{eV}, 8.9 \mathrm{eV}$, and $7.8 \mathrm{eV})$.

(2) The photoelectric efficiencies of the individual dust grains, defined as the number of electrons emitted per photon incident, were measured by direct measurements of the two quantities involved. The photoelectric yields, defined as electrons emitted per photon absorbed, are then determined by convolving the measured photoelectric efficiencies at grain surface potentials approaching zero, with the absorption efficiencies calculated by using Mie theory for the effective radii of the dust grains with assumed complex refractive indices of the grain material. The calculated yields corresponding to any measured values of refractive indices may be conveniently determined in accordance with equation (9), with similar trends and size dependences as discussed in this paper.

(3) The photoelectric efficiencies are found to be a function of the particle charge or surface potential. This dependence is observed to be stronger for small grains of submicron 
size radii compared with grains of larger size. A viable theoretical explanation for this trend remains to be developed.

(4) The measured photoelectric efficiencies and yields of individual lunar dust grains indicate a size dependence with the yields becoming smaller by an order of magnitude for grains with effective radii less than a micron. For larger grains, the yields approach a constant asymptotic value that may be compared with the values obtained from measurements on bulk materials. The asymptotic values, however, are found to be more than an order of magnitude larger than the bulk measurements on lunar fines reported in the literature. The reasons for the discrepancy between the two measurements are not clear.

(5) The yields for the dust grains from the Apollo 17 samples are found to be similar to those measured for the Luna 24 dust grains. The yields for the dust grains selected from the JSC-1 lunar simulants, however, are measured to be lower than the lunar dust grains by factors of the order of 4 to 1.5 at $\mathrm{UV}$ wavelengths of $120 \mathrm{~nm}$ to $160 \mathrm{~nm}$ respectively.

\section{Acknowledgements:}

This work was supported by the Science Directorate at NASA Marshall Space Flight Center under the IRAD program. The Luna 24 and Apollo 17 lunar dust grains employed in this study were provided by Larry Taylor of the University of Tennessee. We are grateful to: Frank Six, John Davis, and Ron Koczor for support and encouragement during the course of this work, and to C. Gallo and V. Kresin for useful information and helpful discussions. We thank C. Ryland and V. Coffey for assistance in the laboratory set up. 


\section{REFERENCES}

Abbas, M. M., Craven, P. D., Spann, J. F., West, E, Pratico, J, Tankosic, D. and Venturini, C.C., 2002, Photoemission Experiments for Charge Characteristics of Individual Dust Grains, Pysica Scripta, T98, 99-103.

Abbas, M. M., Craven,P.D., Spann, J. F., Witherow, W.K., West, E.A., Gallagher, D.L., Adrian, M. L. „Fishman, M. L., Tankosic, D., LeClair, A., Sheldon, R. and Thomas Jr., E., 2003, Radiation pressure measurements on micron-size individual dust grains, Journal of Geophysical. Research, 108, 1229.

Abbas, M. M., Craven, P. D., Spann, J. F., Tankosic, D., LeClair, A., Gallagher, D. L., West, E.A., Weingartner, J. C. , Witherow, J. C. and Tielens, A. G. G. M., 2004, Laboratory Experiments on Rotation and Alignment of the Analogs of Interstellar Dust Grains by Radiation, Astrophysical Journal, 614, 781-795.

Abbas, M. M., Tankosic,D., Craven, P.D., Spann, J. F., LeClair, A., West, E. A.,Weingartner, J. C., Tielens, A. G. G. M., Nuth, J.A., Camata R.P. and Gerakines , P.A., 2006a, submitted for publication.

Abbas, M.M., Tankosic, D., Craven, P.D., Hoover, R.B., Taylor, L.A., Spann, J. F., LeClair, A., and West, E. A., 2006b, Measurements of Photoelectric Yield and Physical Properties of Individual Lunar Dust Grains Proc. Dust in Plan. Sys., Kuai, HI.

Abbas, M.M., Tankosic, D., Craven, P.D., Hoover, R.B., Taylor, L.A., Spann, J. F., LeClair, A., and West, E.A., 2006c, Photoelectric Emission Measurements on Apollo 17 Lunar Dust Grains, $37^{\text {th }}$ Lunar Planet. Sci. Conf., abstract no.1415.

Berg, O.E., 1978, A lunar terminator configuration, Earth and Planetary Science Letters, 39, 377-381

Bohren, C.F. and Huffman, D. R., 1983, Absorption and scattering of light by small particles, John Wiley \& Sons.

Bréchignac, C., Ph. Cahuzac, F. Carlier, M. de Frutos, J. Leygnier, J. Ph. Roux and A. Sarfati, 1992, Ionization vs. neutralization in alkali-atom clusters, Journal of Physics.II France 2, 971-983.

Burtscher, H., and A. Schmidt-Ott., 1982, Enormous Enhancement of van der Waals Forces between Small Silver Particles, Physical Review. Letters. 48, 1734-1737.

Burtscher, H., A. Schmidt-Ott, and H. Siegmann, 1984, Photoelectron yield of small silver and gold particles suspended in gas up to a photon energy of $10 \mathrm{eV}$, Zeitschrift für Physik B Condensed Matter, 56, 197.

Carrier, III, W.D., 1973, Lunar soil grain size distribution, The Moon, 6, 250-263.

Criswell, D.R., 1973, Horizon-glow and the motion of lunar dust, In: R. J. L. Grard (ed), Photons and Particle Interactions with Surfaces in Space, Dordrecht: Reidel, Publish., 37, 545-556.

Costes, N. C., Farmer, J.E. and George, E. B., Mobility performance of the lunar roving vehicle: terrestrial studies-Apollo 15 results, NASA Technical Report, TR R-401, Washington, D. C., 1972.

Cusack, N.,1958, The Electrical and Magnetic Properties of Solids, Longmans, Green and Co.

Davis, E. J., 1985, Electrodynamic balance stability characteristics and applications to the study of aerocolloidal particles, Langmuir, 1 , 379-387. 
de Heer, W. A., 1993, The physics of simple metal clusters: experimental aspects and simple models, Rev. Mod. Phys., 65, No. 3, 611-676.

Draine, B. T., 1978, Photoelectric heating of interstellar gas Astrophysical Journal Supplement, 36, 595-619.

Draine, B.T., Lee, H.M., 1984, Optical properties of interstellar graphite and silicate grains, Astrophysical Journal., 285, 89-108.

Dugourd, Ph., Rayane, D., Labastie, P., Vezin, B., Chevaleyre, J., Broyer, M., 1992, Measurements of lithium cluster ionization potentials, Chemical Physics Letters, 197(45),433-437.

Ekardt, W., 1985, Size-dependent photoabsorption and photoemission of small metal particles, Physical Review B (Condensed Matter), 31(10), 6360-6370.

Freeman, J. W., Jr., Fenner, M. A. and Hills, H. K., The Electric Potential of the Moon in the Solar Wind, In: R. J. L. Grard (ed), Photons and Particle Interactions with Surfaces in Space, Dordrecht: Reidel, Publish., 37, 363

Feuerbacher, B., Annderegg, M. Fitton, B., Laude, L. D.,Willis, R. F. and Grard, R. J. L., 1972, Secondary electron emission characteristics of lunar surface fines, Supplement J. Geochemica et Cosmochimica Acta, 3, 2655.

Gallo, C. F., and Lama, W. L., 1974, Electrostatic models for the correlation between the wotk function and the inozation energy, Institute of Electrical and Electronics EngineersTransactions. On Industry. Applications, 1A-10(4), 496-500.

Gallo, C. F., and Lama, W. L., 1976a, Classical electrostatic description of th work function and ionization energy of insulators Institute of Electrical and Electronics EngineersTransactions. On Industry. Applications, 1A-12(1), 7-11.

Gallo, C. F., and Lama, W. L., 1976b, Some charge exchange phenomena explained by a classical model, Journal of Electrostatics, 2. 145-150.

Heiken, G. H., Vaniman, D. T. and French, B. M., 1991, Eds., Lunar Source Book, Cambridge University Press.

Halekas, J. S.; Mitchell, D. L.; Lin, R. P.; Hood, L. L.; Acuña, M. H.; Binder, A. B., 2002, Evidence for negative charging of the lunar surface in shadow, Geophysical Research Letters, 29(10), 1435-1439.

Horanyi, M., Robertson, S. and Walch, B., 1995, Electrostatic charging properties of simulated lunar dust, Geophysical Research Letters, 22, 2079-2082.

Horanyi, M., 1996, Charged Dust Dynamics in the Solar System, Annual Review of Astronomy and Astrophysics, 34, 383-418.

Horanyi, M., B. Walch , Robertson, S. and Alexander, A., 1998, Electrostatic charging properties of Apollo 17 lunar dust, Journal of Geophysical Research, 103, 8575-8580.

Johnson, S. W., Taylor, G. J. and Wetzel, J. P., 1992, Environmental effects on lunar astronomical observatories, $2^{\text {nd }}$ Conf. On Lunar Bases \& Space Activities, 1, 329-335.

Kittel, C., 1996, Introduction to Solid State Physics, $7^{\text {th }}$ ed., Wiley, New York.

Manka, R. H., 1973, Plasma and Potential at the Lunar Surface, In: R. J. L. Grard (ed), Photons and Particle Interactions with Surfaces in Space, Dordrecht: Reidel, Publish., 37, 347-361.

McCoy, J.E., and Criswell, D.R., 1974, Evidence for a high altitude distribution of lunar dust, Proc. $5^{\text {th }}$ Lunar Conference, 3, 2991-3005.

McKay, D. S., R. M. Fruland, and G. H. Heiken, 1974, Grain size and the evolution of lunar soils, Geochimica. et Cosmochimica Acta, 1, 887-906. 
Müller, U., Schmidt, A. and Burtscher H., 1988, Photoelectric quantum yield of free silver particles near threshold, Zeitschrift für Physik B Condensed Matter, 73, 103.

Pelizzari, M. A., and D. R. Criswell, 1978, Lunar dust transport by photoelectric charging at sunset, Proc. Lunar Planet. Sci. Conf., $9^{\text {th }}$, 3225-3237.

Rennilson, J. J., and Ciswell, D. R.,1974, Surveyor observations of Lunar horizon-glow, The Moon, 10, 121-142.

Rose, H. J., Jr.; Baedecker, P. A., Berman, S.,Christian, R. P., Dwornik, E. J.,Finkelman, R. B.; Schnepfe, M. M., 1975, Chemical composition of rocks and soils returned by the Apollo 15, 16, and 17 missions, In: , Proceedings of the 6th Lunar Science Conference, 6th, New York, Pergamon Press, Inc.,2, 1363-1373.

Schmidt-Ott, A., P. Shurtenbrger, and H. C. Siegmann, 1980, Enormous yield of photoelectrons from small particles, Physi. Rev. Lett., 45, 1284-1287.

Seidel, M. and Brack, M., Finite-size effects in ionization potentials and electron affinities of metal clusters, The Journal of Chemical Physics ,95(2), 1295-1303, 1991.

Spann, J.F., Abbas, M.M., Venturini, C. C. and Comfort, R.H. 2001, Electodynamic Balance for studies of cosmic dust paricles Physica Scripta., T89, 147-153.

Stubbs, T.J., Vondrak, R. R., Farrell, W. M., 2006, A dynamic fountain model for lunar dust, Advances in Space Research, 37(1), 59-66.

Watson, W. D., 1972, Heating of Interstellar H i Clouds by Ultraviolet Photoelectron Emission from Grains, Astophysical Journal., 176, 103.

Watson, W. D., 1973, Photoelectron emission from small spherical particles Journal of the Optical Society of America, 63, 164.

Weingartner, J. C., and Draine, B. T. 2001, Photoelectric emission from interstellar dust: Grain charging and gas heating, ApJ. Supp., 134, 263.

Wiscombe, W. J., 1979, Mie Scattering Calculations: Advances in Technique and Fast Vector Speed Computer Codes, Document PB301388, NTIS, Springfield, VA 22161.

Wong, K., S. Vongehr, and V. V. Kresin, 2003, Work functions, ionization potentials, and in between: Scaling relations based on the image-charge model, Physical Review B, 67, 035406, 2003.

Zook, H. A., and McCoy, E., 1991, Large scale lunar horizon glow and a high altitude lunar dust exosphere, Geophysical Research Letters, 18, 2117.

Zook, H. A.; Potter, A. E.; Cooper, B. L., 1995, The Lunar Dust Exosphere and Clementine Lunar Horizon Glow, Abstracts of the Lunar and Planetary Science Conference, 26, 15771578. 


\section{LIST OF FIGURES}

Figure 1. A schematic of the setup of an electrodynamic balance for UV photoelectric yield measurements.

Figure 2. A polystyrene particle of $2.3 \mu \mathrm{m}$ radius successively charged with UV radiation, discharged with a $2500 \mathrm{eV}$ electron beam, and charged with "white light" UV radiation from deuterium lamp.

Figure 3. Variation of charge $q$ and DC voltage $V_{D C}$ of a $0.18 \mu \mathrm{m}$ radius negatively charged Apollo 17 dust grain exposed to UV radiation. The particle is ejected from the trap when the charge approaches zero.

Figure 4. The absorption efficiency $Q_{a b s}$ as a function of the size parameter $(2 \pi \mathrm{a} / \lambda)$, using Mie scattering theory, using complex refractive indices for Silica from the data in Draine and Lee (1984).

Figures 5a-c. Plots of the photoelectric efficiencies of two individual Apollo 17 dust grains of effective radii $0.18 \mu \mathrm{m}$ and $1.75 \mu \mathrm{m}$ radii illuminated with $U V$ radiation at 120,140 , and 160 nm wavelengths.

Figure 6. A composite plot of the photoelectric yields $\mathrm{Y}$ of about 10 dust grains of Apollo 17 return samples of effective radii in the range of $0.18 \mu \mathrm{m}$ to $11.78 \mu \mathrm{m}$ at $\mathrm{UV}$ wavelengths of 120,140 , and $160 \mathrm{~nm}(10.3,8.9$, and $7.9 \mathrm{eV})$ as a function of the size-parameter $x=(2 \pi a / \lambda)$. Measurements on bulk lunar materials by Feuerbacher et. al. (1972), are also shown for comparison.

Figures 7a-b. Plots of the photoelectric efficiencies of two individual Luna-24 dust grains of effective radii $0.77 \mu \mathrm{m}$ and $3.38 \mu \mathrm{m}$ radii illuminated with UV radiation at 120,140 , and 160 nm wavelengths. The details are as in Fig. 5.

Figure 8. A composite plot of the photoelectric yields $\mathrm{Y}$ of 17 dust grains of Luna-24 return samples of effective radii in the range of $0.10 \mu \mathrm{m}$ to $11.75 \mu \mathrm{m}$. The details are as in Fig. 6 .

Figures 9a-b. Plots of the photoelectric efficiencies of two individual JSC-1 Simulant dust grains of effective radii $0.70 \mu \mathrm{m}$ and $3.25 \mu \mathrm{m}$ radii illuminated with UV radiation at 120,140 , and $160 \mathrm{~nm}$ wavelengths. The details are as in Fig. 5.

Figure 10. A composite plot on the photoelectric yields Y of 8 dust grains from JSC-1 simulant grains of effective radii in the range of $0.17 \mu \mathrm{m}$ to $4.6 \mu \mathrm{m}$. The details are as in Fig. 6. 
Fig.1
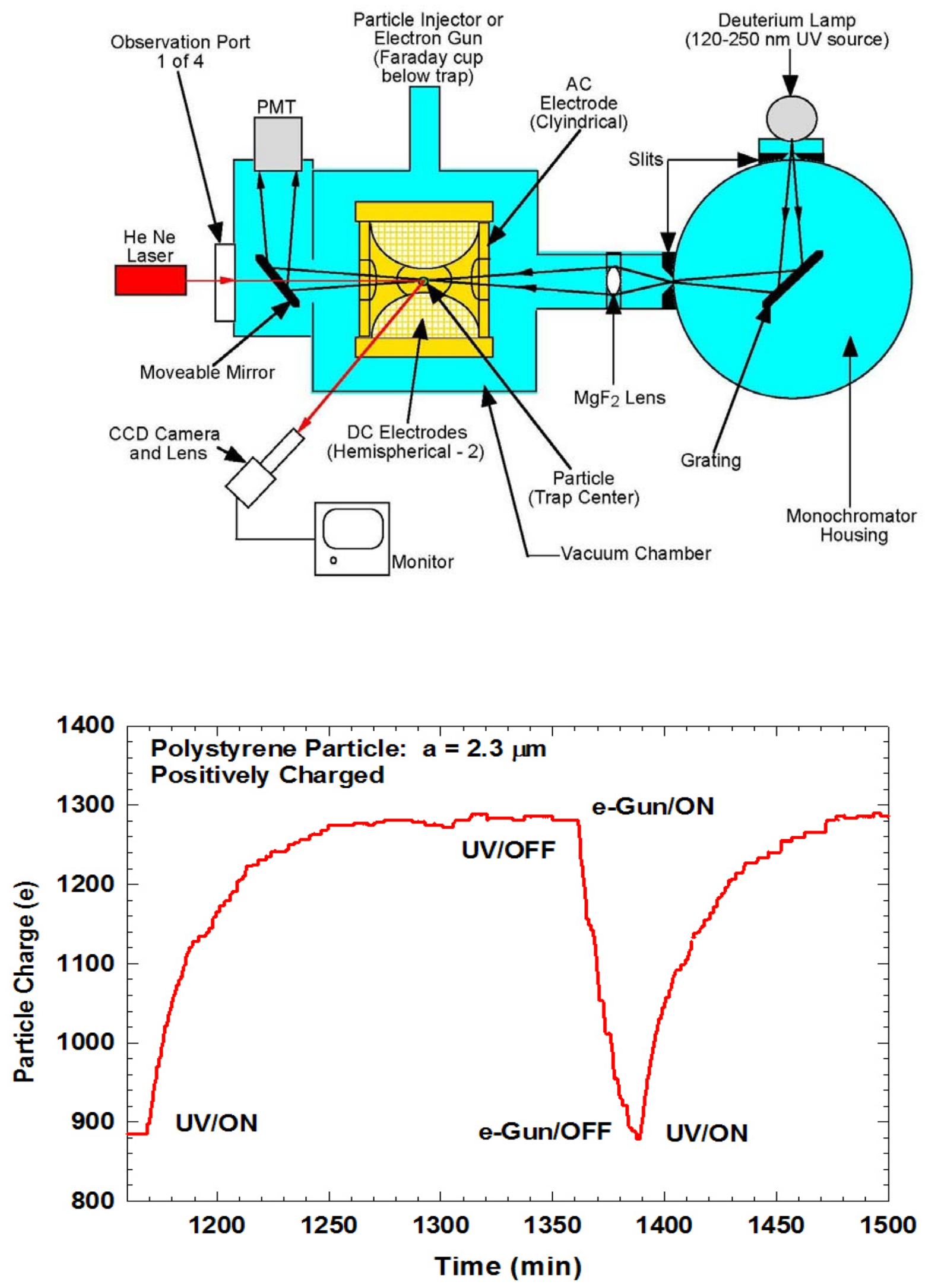

Fig.2 


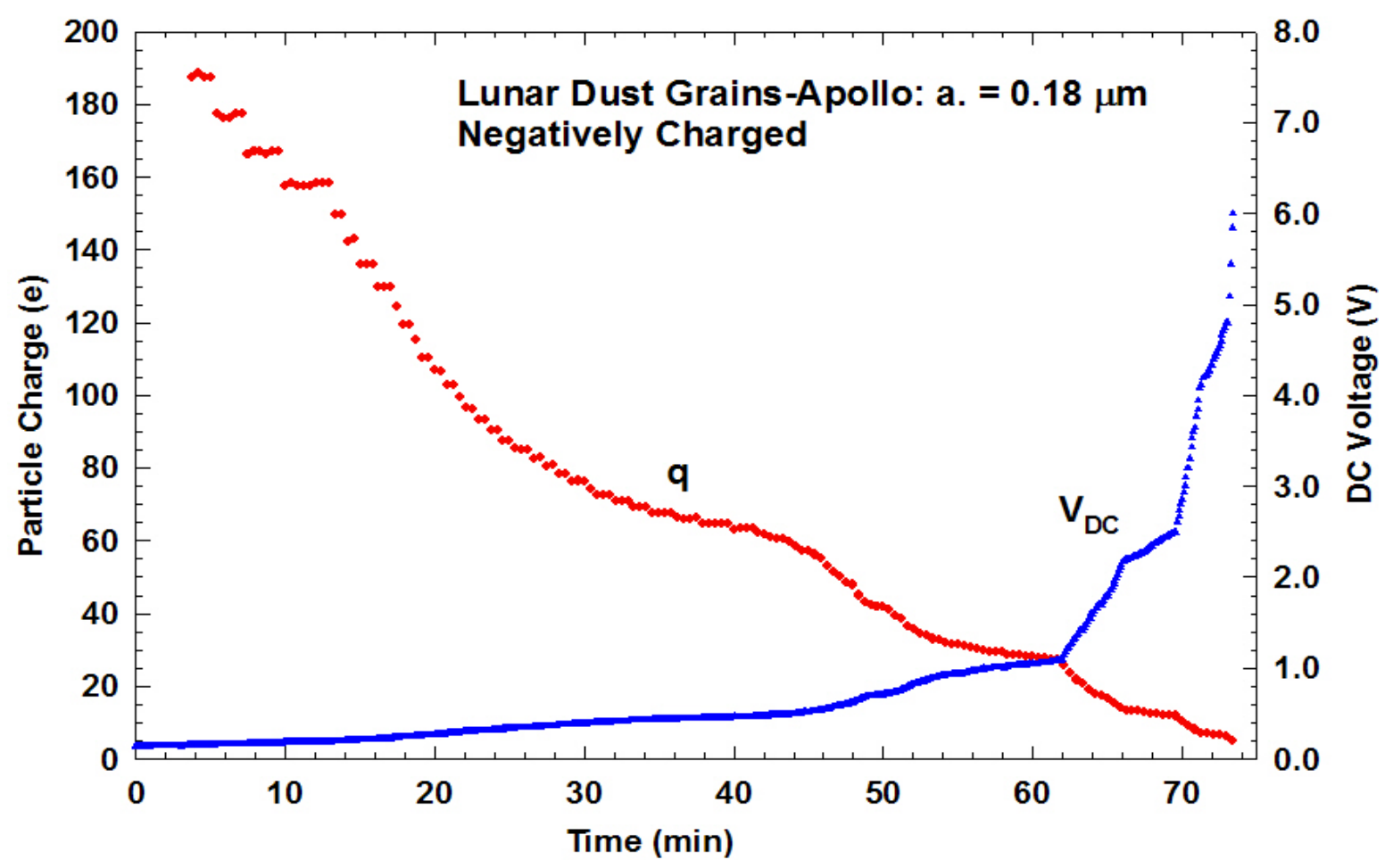

Fig. 3

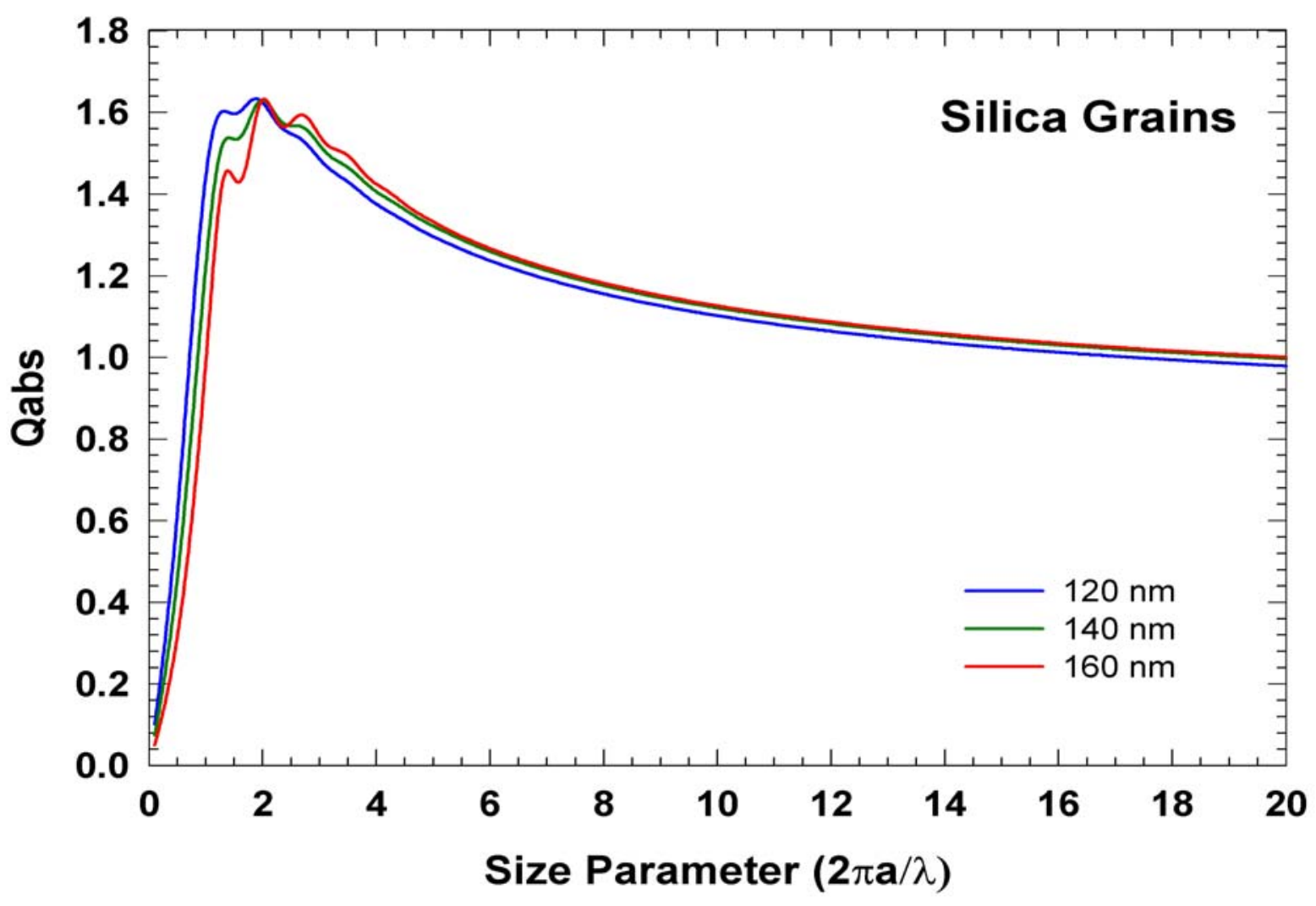

Fig. 4 


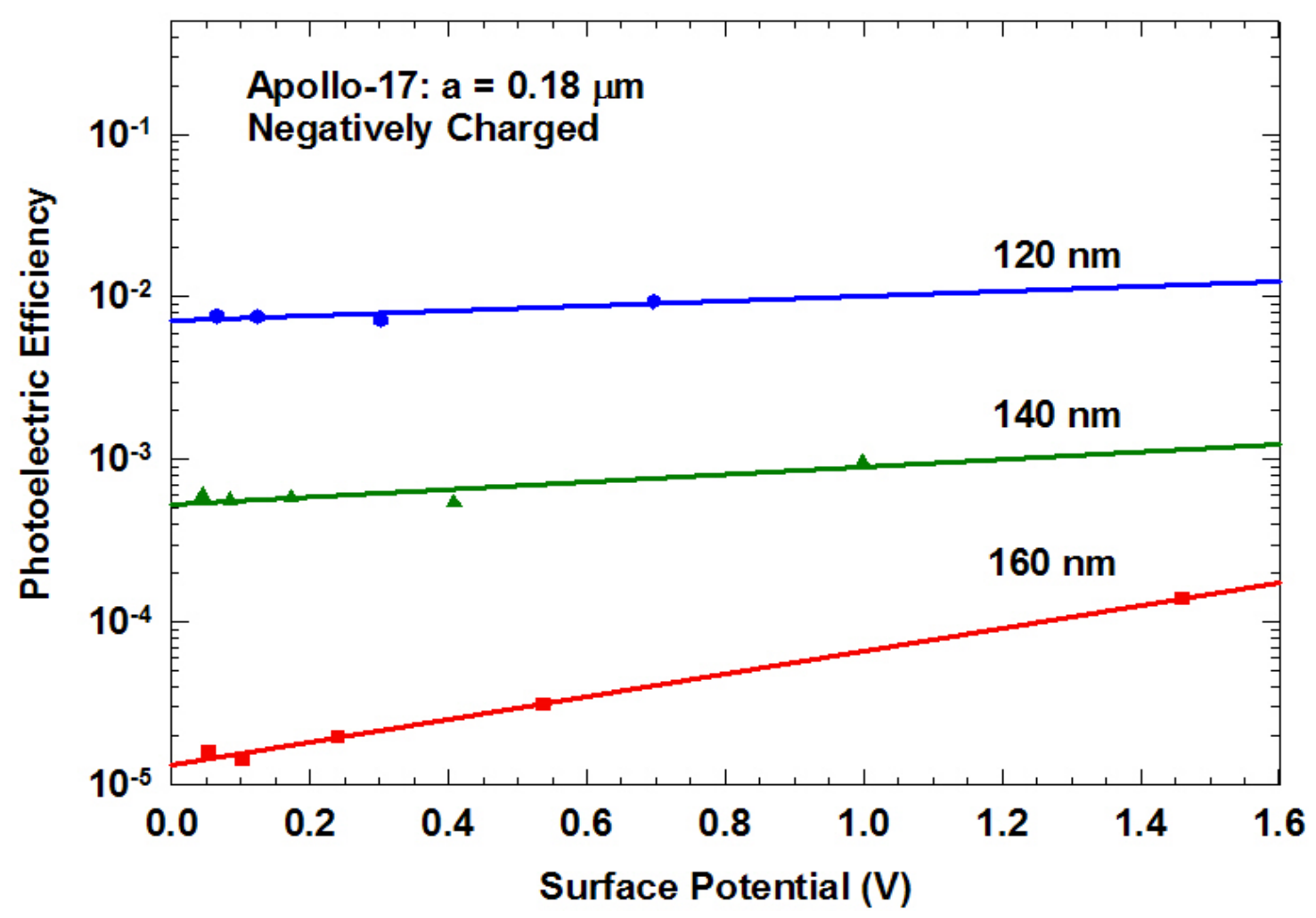

Fig. 5a

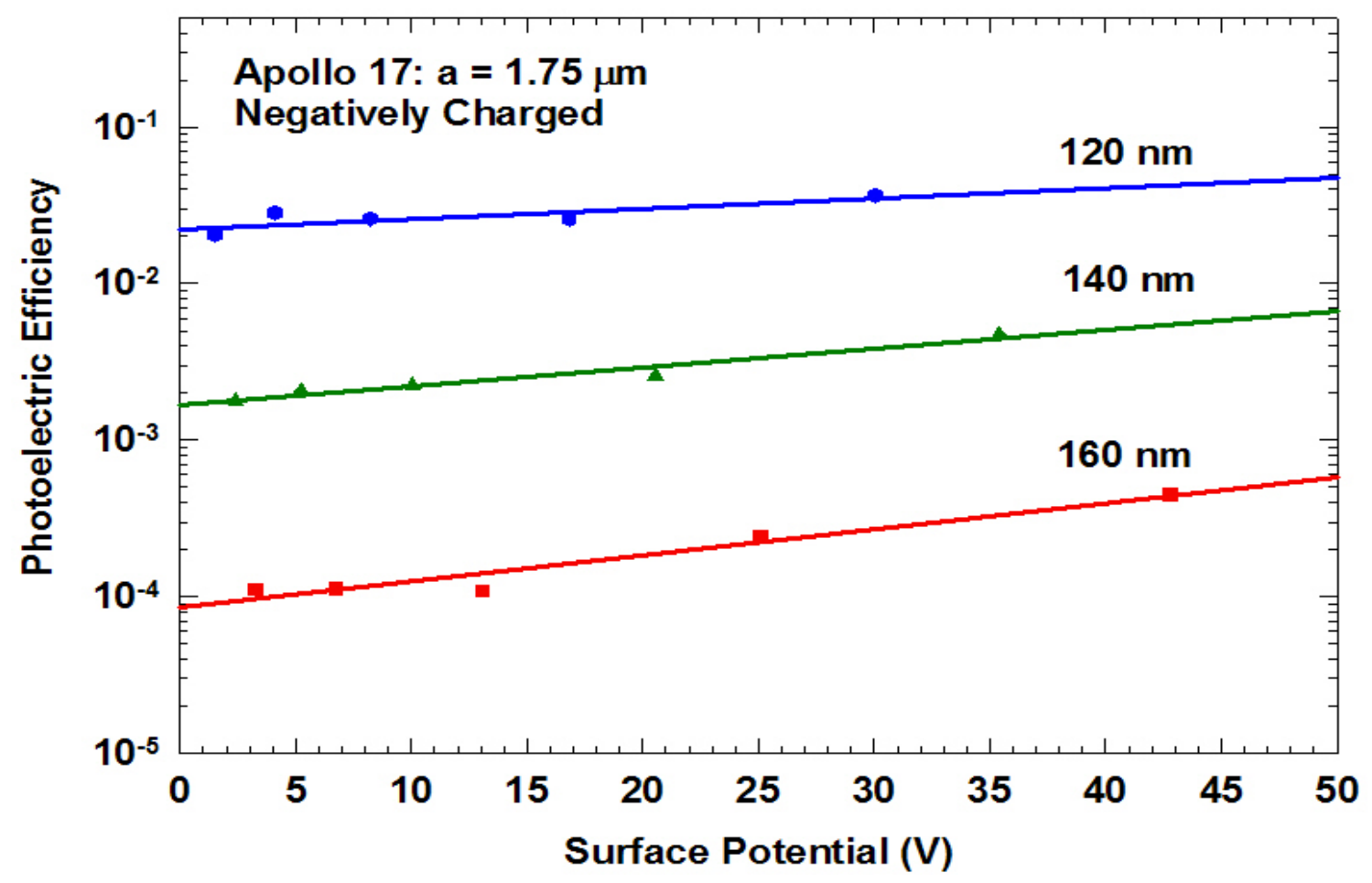

Fig. 5b 


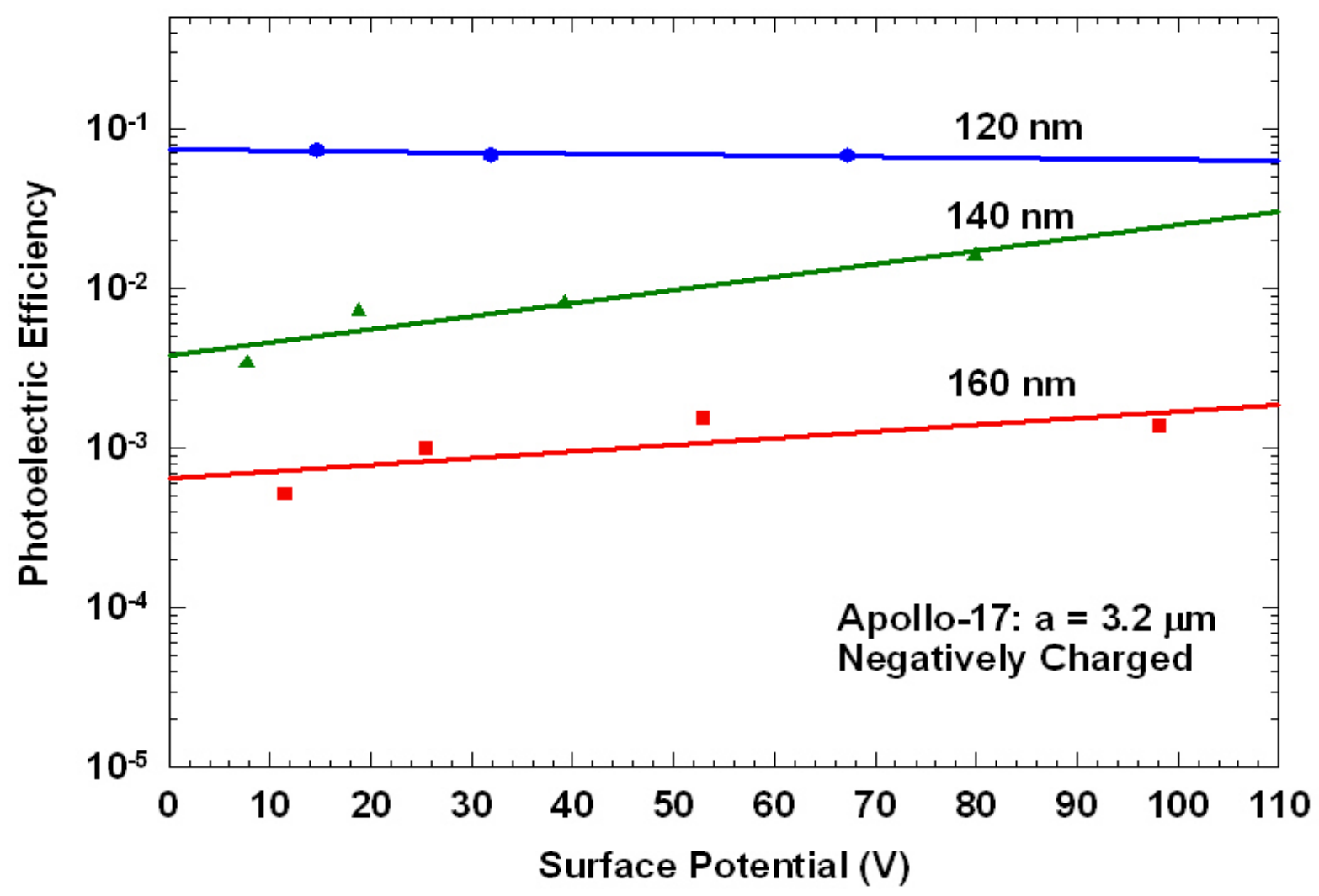

Fig. 5c 


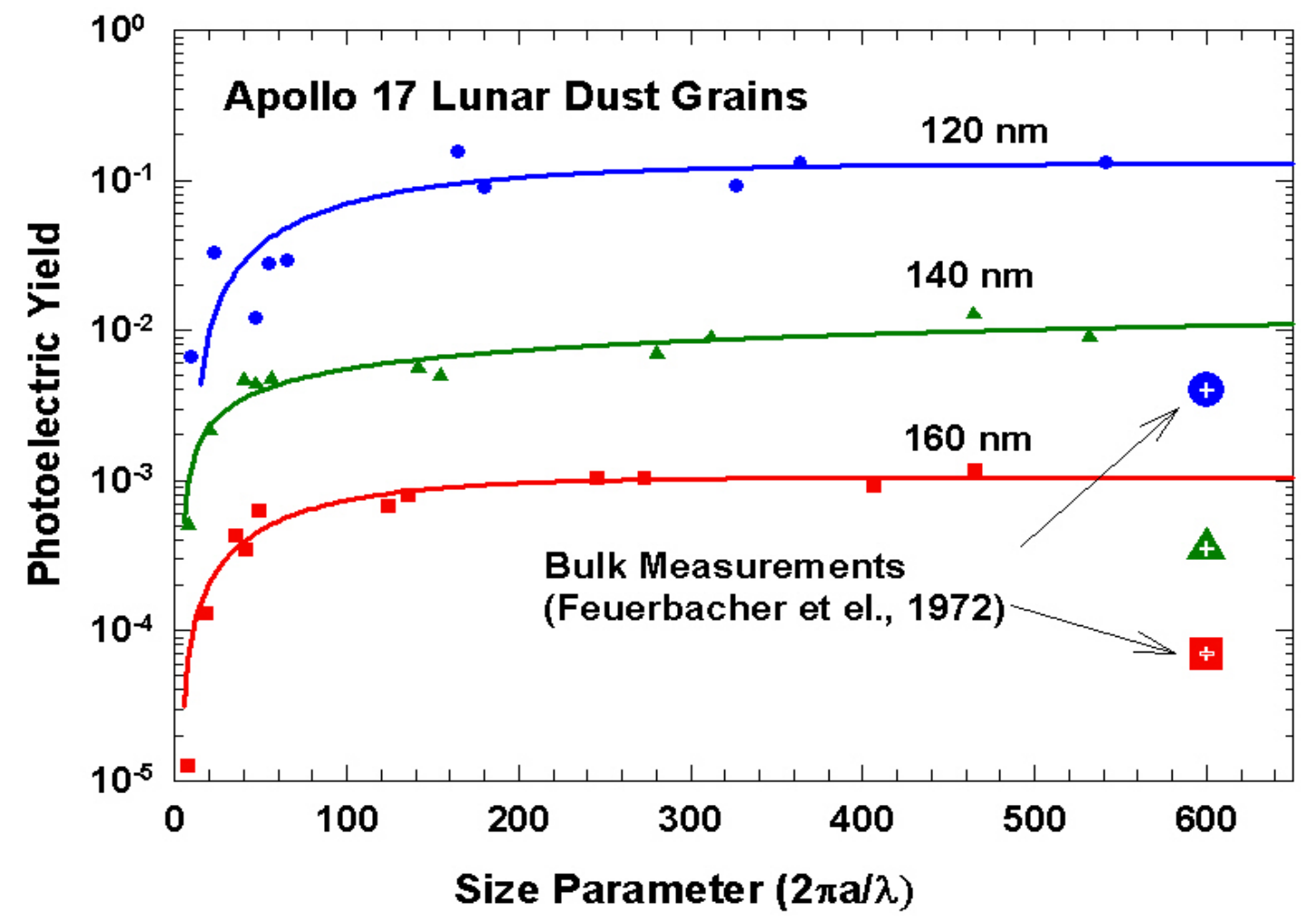

Fig. 6 


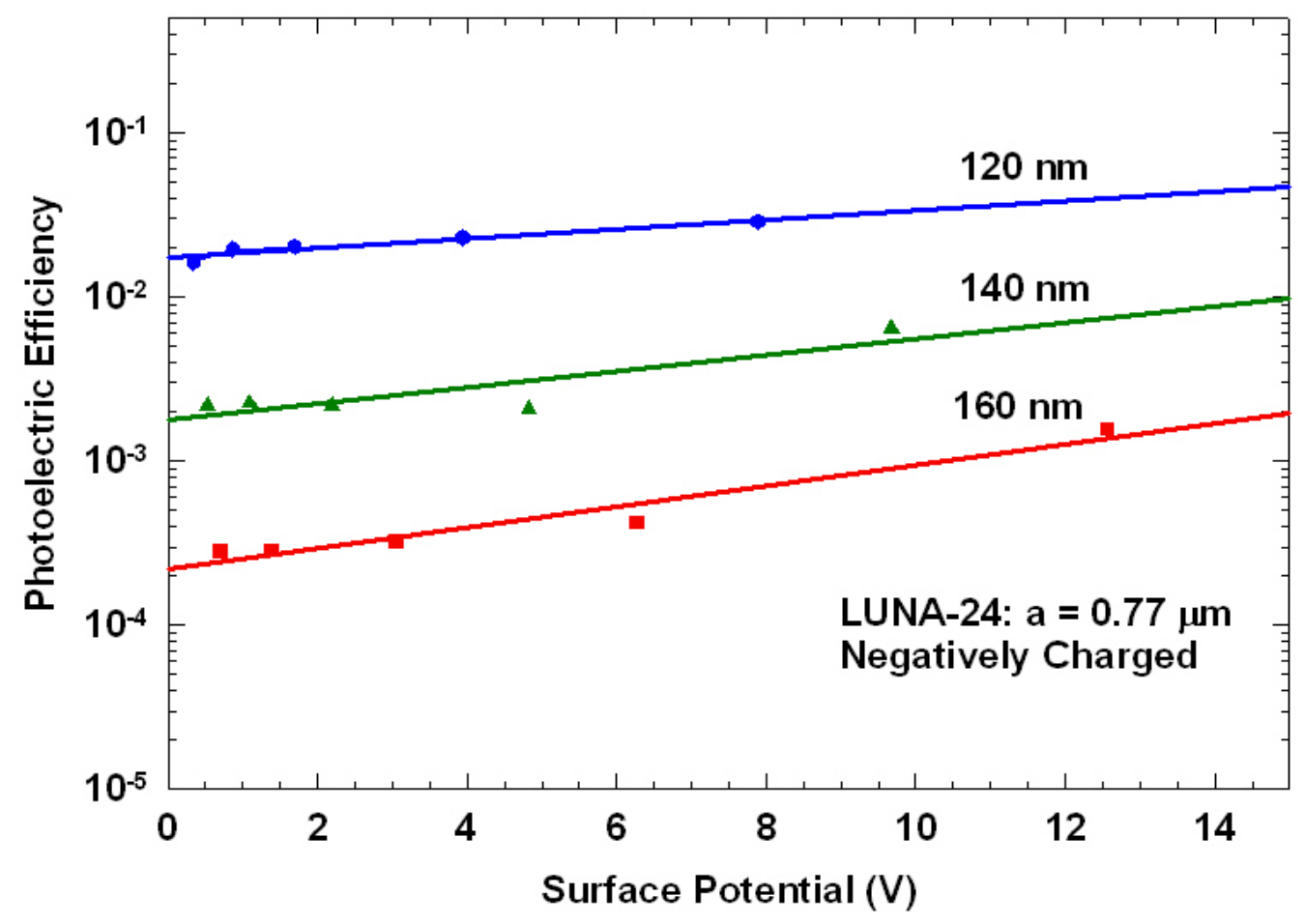

Fig. 7a

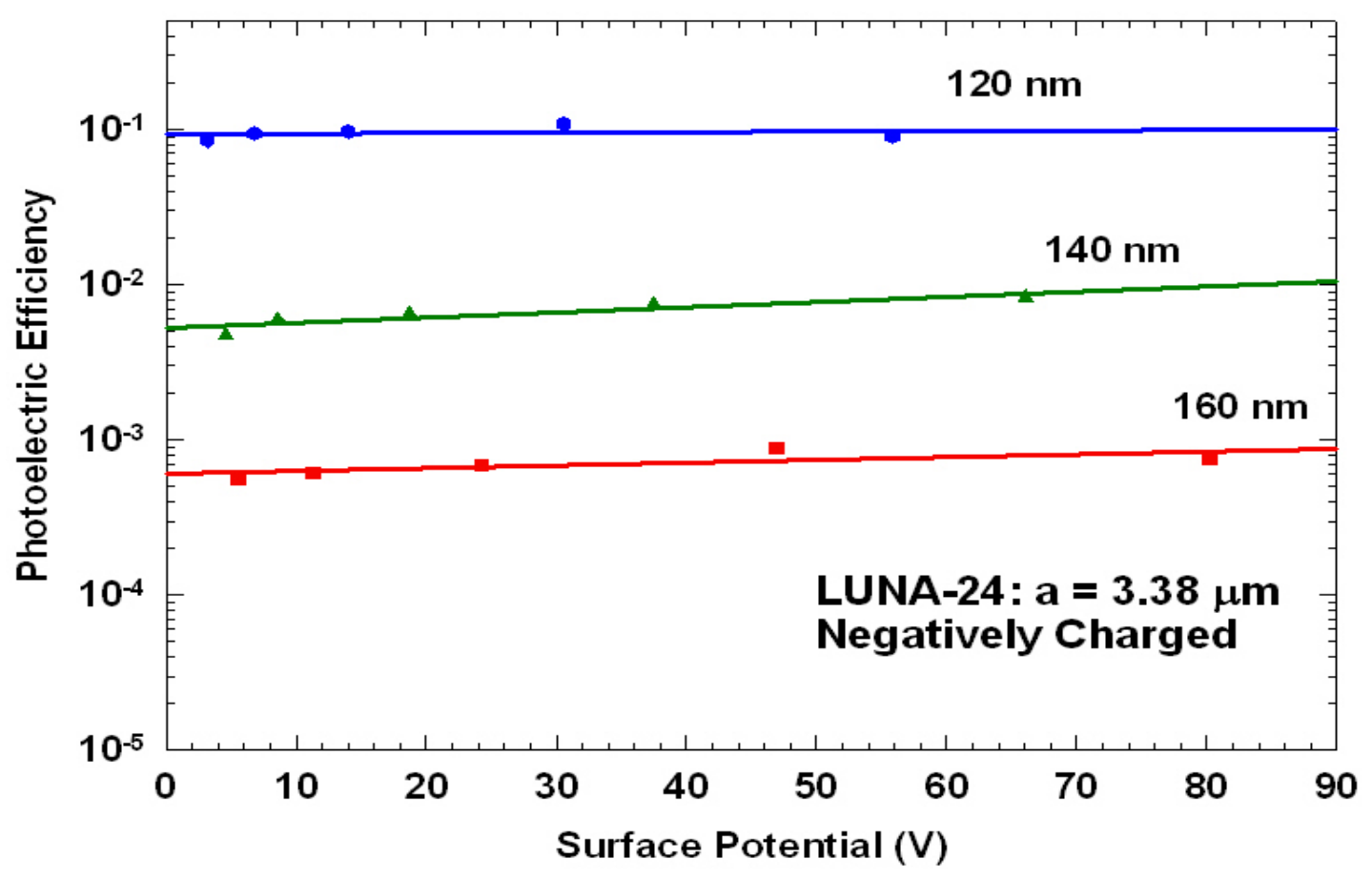

Fig. 7b 


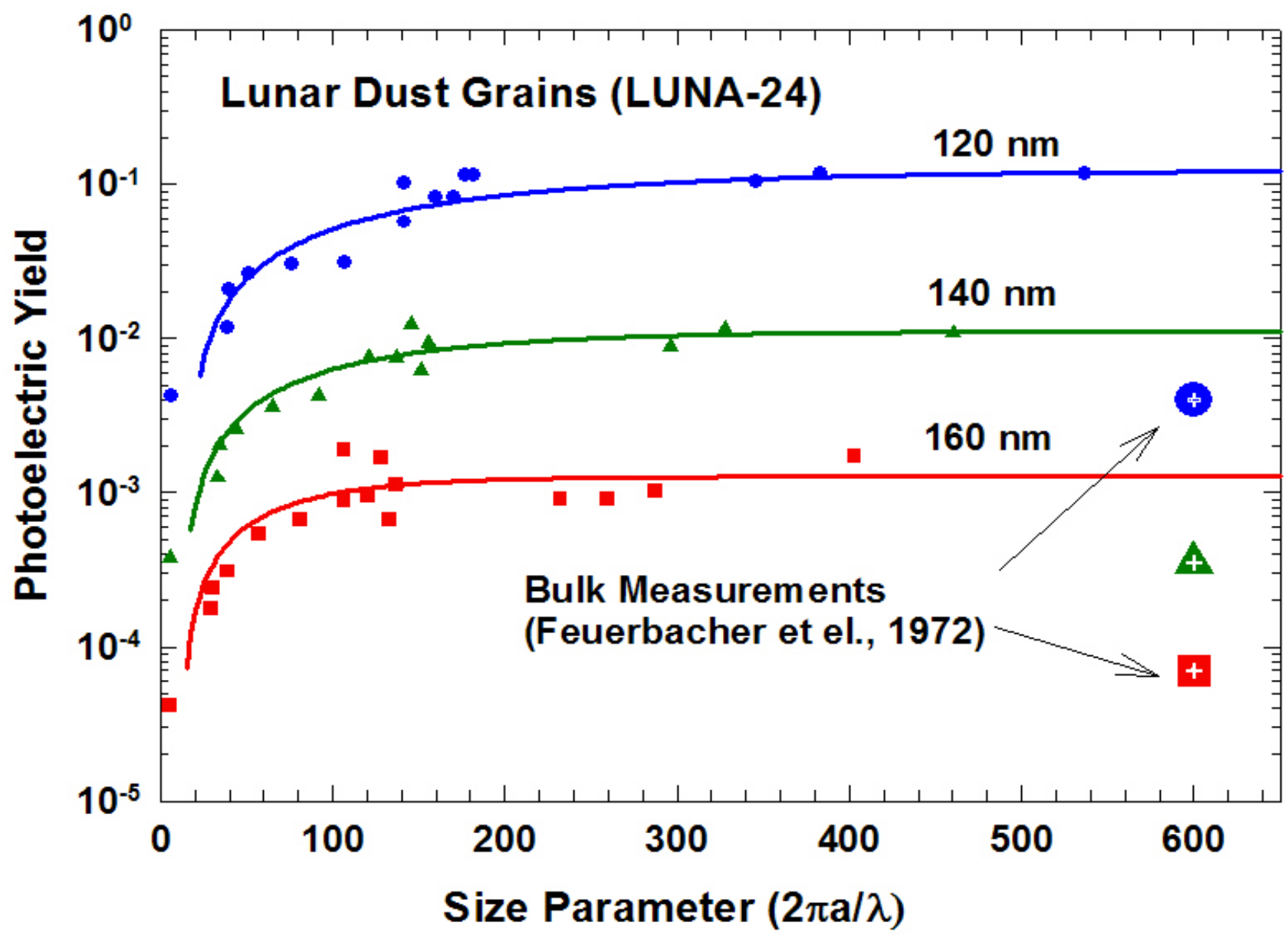

Fig. 8 


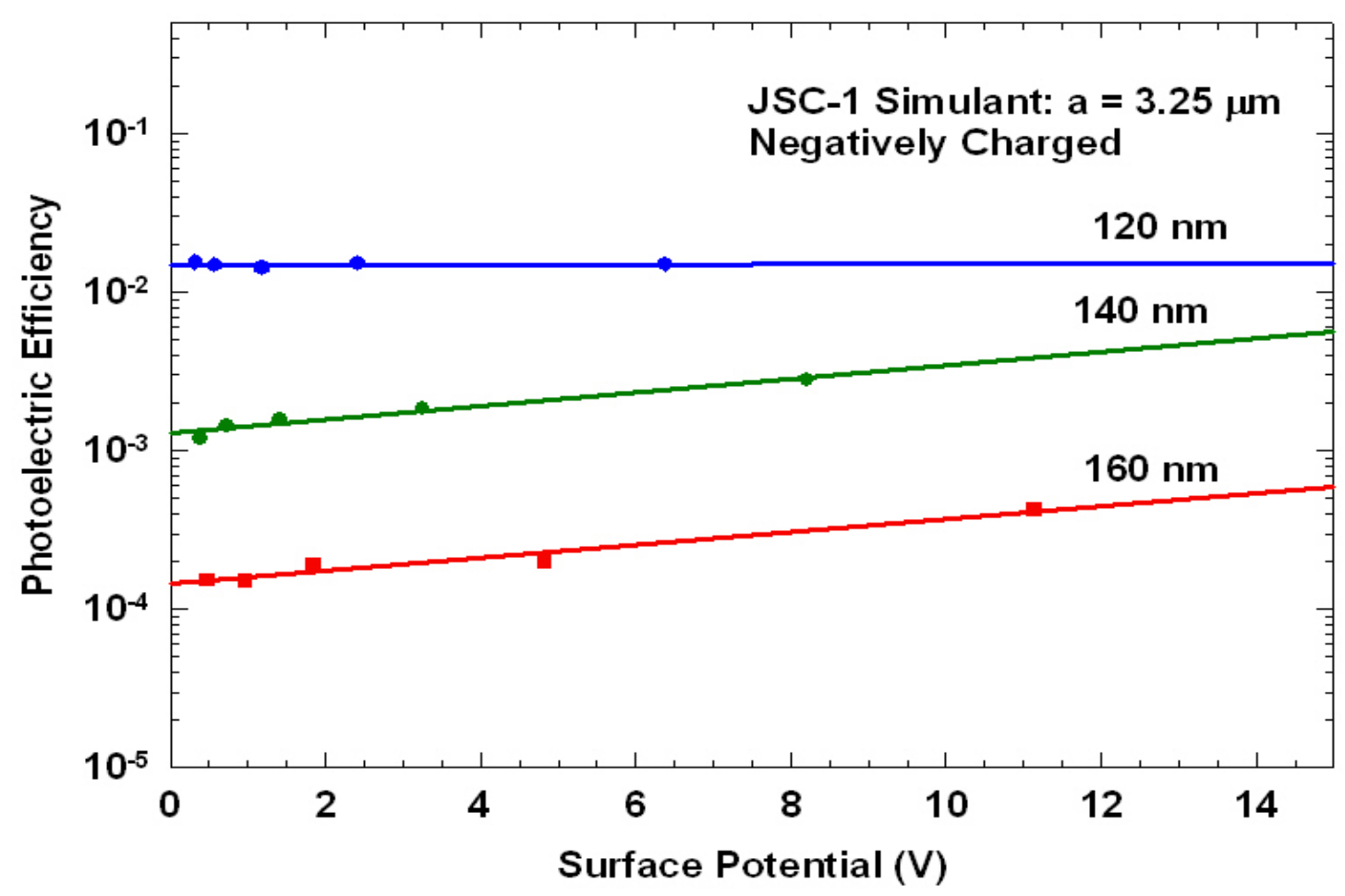

Fig. 9a

Fig. 9b

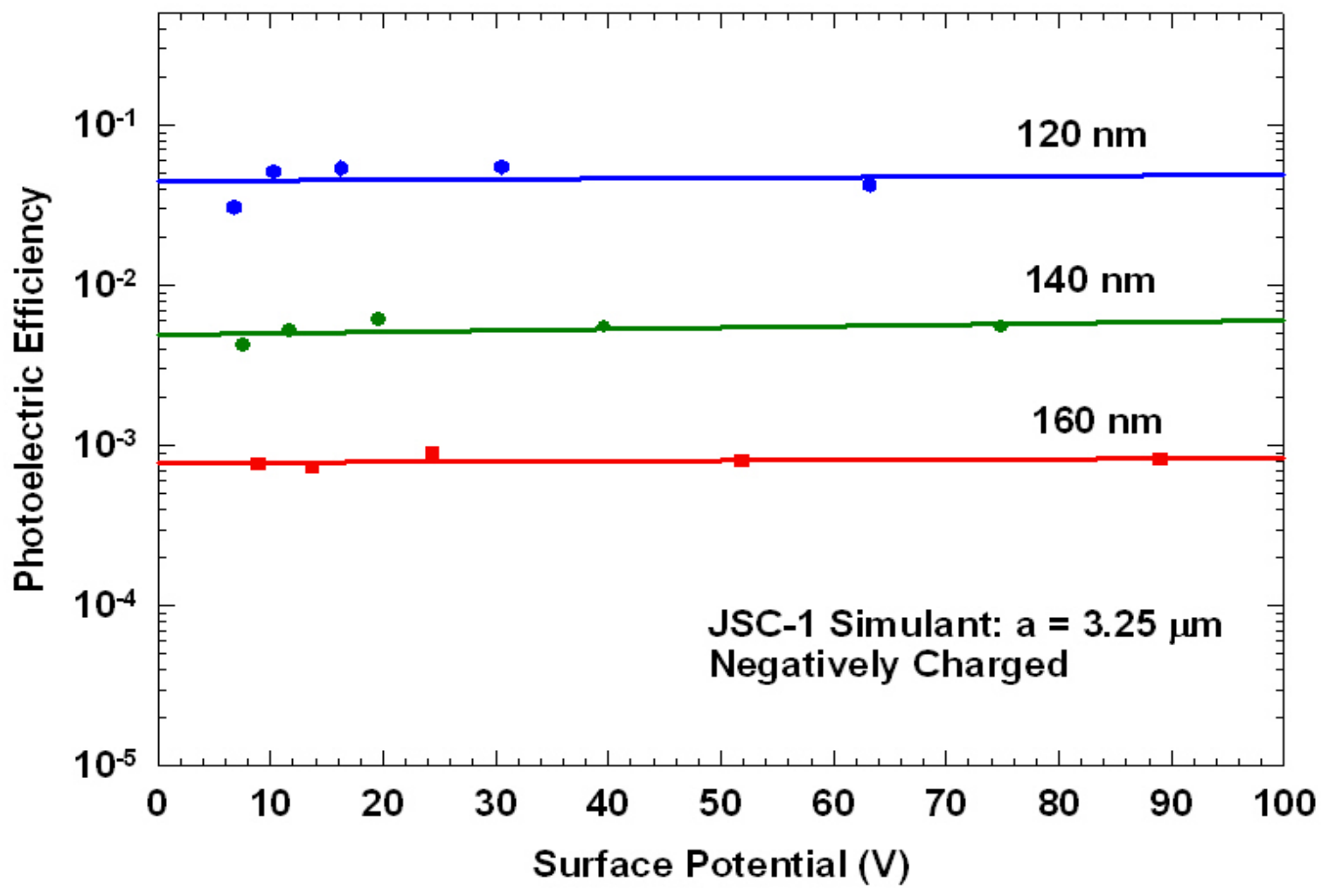




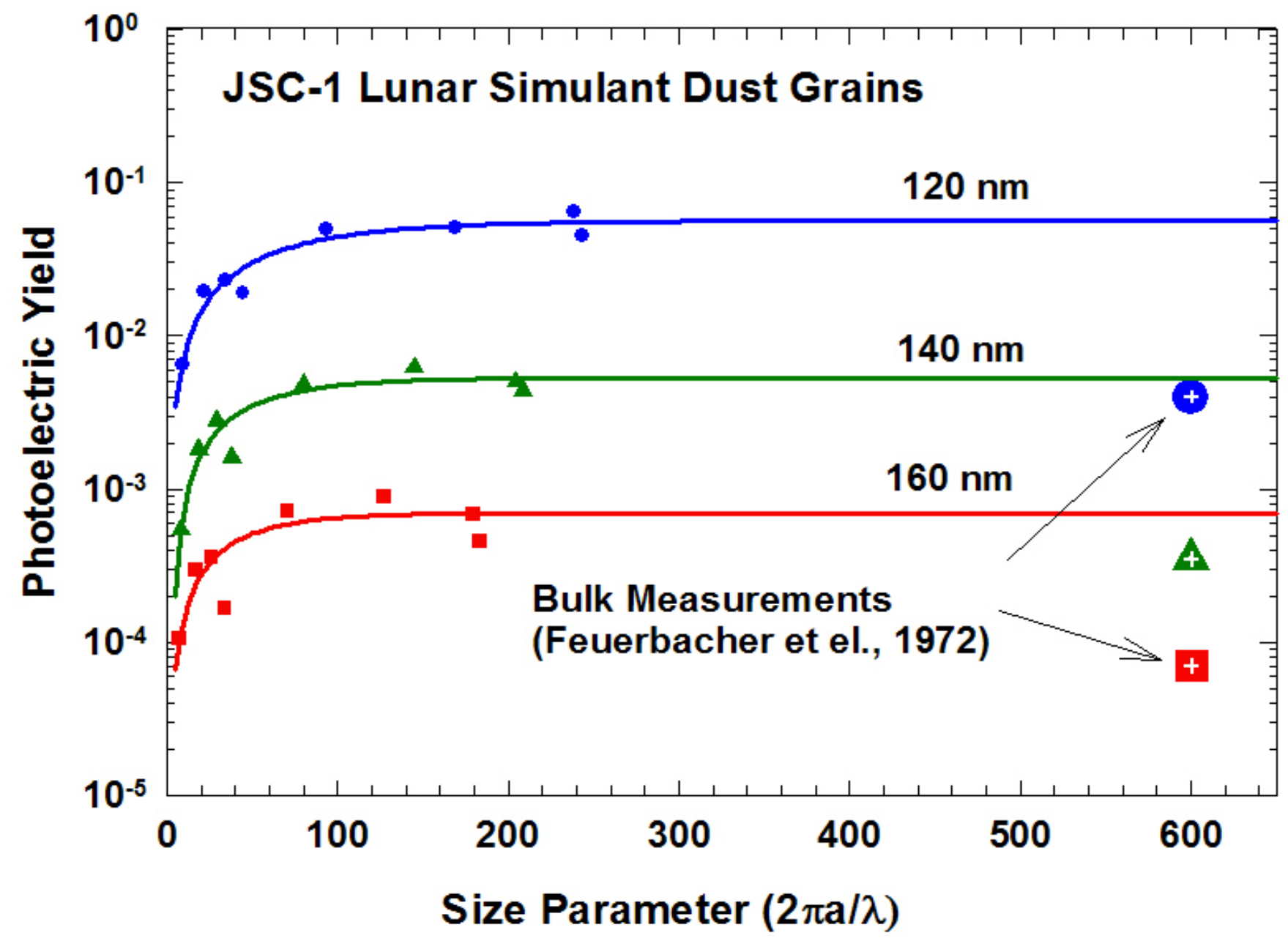

Fig. 10 